\title{
PERCEPCIÓN DE JÓVENES ESCOLARIZADOS SOBRE EL ÉXODO \\ DE MIGRANTES VENEZOLANOS: ANÁLISIS DE LAS ACTITUDES XENOFÓBICAS EN CONTEXTOS FRONTERIZOS DESDE LO SUTIL Y LO MANIFIESTO
}

\section{PERCEPTION OF YOUNG SCHOOLED ON THE EXODUS OF VENEZUELAN MIGRANTS: ANALYSIS OF XENOPHOBIC ATTITUDES IN BORDER CONTEXTS FROM THE SUBTLE AND THE MANIFESTO}

\author{
Audin Alioso Gamboa Suárez ${ }^{1}$ \\ César Augusto Hernández Suárez² \\ William Rodrigo Avendaño Castro ${ }^{3}$
}

Colombia

1 Doctor en Ciencias de la Educación por la Universidad de Cartagena (Colombia). Docente investigador de la Universidad Francisco de Paula Santander. Correo electrónico: audingamboa@ufps.edu.co. Orcid: 0000-0001-9755-6408.

$2 \quad$ Magister en Educación Matemática por la Universidad Nacional Experimental del Táchira (Venezuela). Docente investigador de la Universidad Francisco de Paula Santander. Correo electrónico: cesaraugusto@ufps.edu.co. Orcid: 0000-00027974-5560. dad Francisco de Paula Santander. Correo electrónico: williamavendano@ufps.edu.co. Orcid: 0000-0002-7510-8222. 


\section{RESUMEN}

El presente artículo de investigación aborda un fenómeno que durante los últimos años ha generado preocupación entre investigadores en el campo de las Ciencias Sociales y Humanas debido al masivo éxodo de población venezolana al territorio colombiano. Por esta razón, el texto pretende mostrar las percepciones que tienen jóvenes escolarizados sobre la migración desde el prejuicio sutil y manifiesto a este tipo de población. El estudio se aborda desde el paradigma cuantitativo - descriptivo. Se aplicó una encuesta a 616 adolescentes matriculadas en los grados Décimo y Undécimo durante el año 2020 en diversas instituciones educativas de un municipio fronterizo con Venezuela. Los resultados muestran que se evidencia un rechazo radical para entablar relaciones cercanas hacia la población migrante en el contexto social, familiar y laboral.

\section{PALABRAS CLAVE:}

migración, prejuicio manifiesto, prejuicio sutil, xenofobia

\section{ABSTRACT}

This research article addresses a phenomenon that in recent years has generated concern among researchers in the field of Social and Human Sciences due to the massive exodus of the Venezuelan population to Colombian territory. For this reason, the text aims to show the perceptions that young people in school have on migration from the subtle and manifest prejudice to this type of population. The study is approached from the quantitative - descriptive paradigm. A survey was applied to 616 adolescents enrolled in the tenth and eleventh grades during the year 2020 in various educational institutions of a border municipality with Venezuela. The results show that there is a radical refusal to establish close relationships with the migrant population in the social, family and work context.

\section{KEYWORDS:}

migration, manifest prejudice, subtle prejudice, xenophobia

\section{INTRODUCCIÓN}

La Xenofobia es considerada como un proceso comunicativo que rechaza y excluye a un grupo nacional o étnico ajeno, es decir, manifiesta un rechazo simbólico o real hacia aquellas personas que no pertenecen a la propia nación. Todo esto, explicado por Chavarría (1994), revela que lo que lleva a expresar esta actitud hacia el extranjero, es un sentimiento de pérdida de la identidad, además de esa búsqueda por remarcar la identidad grupal, de no quedarse a la deriva en la búsqueda de quién soy y con quién estoy, pero, sobre todo, el recelo por la influencia negativa que traiga consigo el extranjero en términos económicos, sociales, políticos y culturales.

\section{La xenofobia contempla numerosas} genealogías simbólicas que van desde el ámbito de la categorización estereotipada del otro hasta una cierta definición de las identidades colectivas, que se suele traducir en una conciencia esencialista de pertenencia. Establece así una analogía entre el sentimiento xenófobo y el nacionalista en tanto que expresiones de un tipo de comunicación intergrupal manifiesta o potencialmente excluyente, basada, sobre todo, en una politización de las identidades culturales y en la creación de fronteras reales o simbólicas (Chavarría, 1994, p. 139).

Otros autores afirman que la xenofobia es una forma de discriminación, que, como otras actitudes que tiende a tomar el ser humano en pro de automatizar más que concientizar y racionalizar para ahorrarse así el entrenar y 
nutrir su sistema cognitivo, se produce en el intento de dar una respuesta a la situación de crisis por medio del fanatismo, un sentimiento de superioridad y la tendencia a estereotipar todo. En concreto, sus causas se pueden estudiar adentrándose en lo que es nacionalismo, aporofobia y el temor hacía lo nuevo (Cisneros, 2001, p.18). Por otro lado, Bouza (2002), recalca el papel central de un "cierre espacial" y la "defensa de la privacidad", aspectos que posee el ser humano según la psicología y que le ayudan, en cierta forma, a encontrar la manera de defender sus derechos y preservar lo que siente como propio. Por su parte, Stavenhagen (1994) menciona, hablando de la xenofobia, aquella protección de la colonización, del maltrato e invasión por poder de otras naciones. En la búsqueda de poder y en la desconfianza por no dejar ser atacado por otros, el hombre, inferioriza otros, desahogando así sus odios y frustraciones.

En este contexto, es necesario señalar que las conductas xenofóbicas no solo surgen por el problema del éxodo venezolano a Colombia; hay múltiples estudios en otros ámbitos que afirman que la xenofobia a nivel general, como "construcción social del otro como el enemigo, aprovisiona los argumentos legales para una política pública que va en contra de los más elementales valores humanos $y$, en muchas ocasiones, violentadora de los derechos humanos" (Gaborit, 2020, p.45); el mismo autor nos menciona dos ejemplos; el caso del ex presidente Trump en EE. UU, cuando con su política caracteriza a migrantes como "narcotraficantes y violadores" (Washington Post, 2015 citado en Gaborit, 2020), y el caso nazi, con una "sobresimplifcación de las características del pueblo judío, por ejemplo, sirvió como base ideológica para buscar su extermino por parte del nazismo" (Gaborit, 2020, p.57). Por otro lado, en España, se percibe aquél sentimiento de invasión ya mencionado, adicionando imaginarios de la migración relacionados con el aumento de la inseguridad ciudadana, competencia laboral, la poca convivencia vecinal, además de que "destrozan" recintos alquilados y se llevan protagonismo en la defensa de sus derechos y ayudas sociales; imaginarios remarcados e influenciados por los medios (D'Ancona \& Valles, 2010).

Otro estudio, realizado en municipios y barrios de Almería, España, devela tres potenciales causas de la xenofobia, afirmando que, además de que el no conocer y el no ser cercanos a los migrantes, hace a las personas nativas más reacias, también existe un factor de densidad y lugar de procedencia. La cantidad de inmigrantes "fomenta las conductas y actitudes xenófobas de la población, tanto de aquellos individuos que viven en municipios como en barrios de la provincia de Almería, así como la diferenciación (segmentación) de la xenofobia atendiendo al lugar de origen (Herranz, 2008).

Dicha idea de influencia xenofóbica desde los medios de comunicación también es vista desde el contexto de discriminación hacia bolivarianos y peruanos, por Fernández (2004), como detonante de prejuicios hacía estos, aunque recalcando el papel principal que tiene la literatura y la formación con respecto a la historia en la que Chile se ve superior a Bolivia y a Perú; literatura y formación con la que periodistas y comentaristas de fútbol generan mensajes xenofóbicos a la vez que publican noticias "vendibles" (Fernández, 2004). Por esto, Cortés (2018), argumenta que existe un gran reto en la educación de los nuevos periodistas entorno a un aspecto crítico donde saque a la luz la verdad por los que aman y se olvide el amarillismo, fanatismo y las calumnias de los que odian.

Preguntarse sobre los métodos de deshumanización discursiva que los periodistas usan en nuestro contexto, sobre cómo los reporteros crean discursivamente las identidades de los migrantes 
y sobre cómo los noticieros construyen su aparente autoridad para representar a los venezolanos puede contribuir a un entendimiento más profundo sobre la manera en que el orientalismo y el cubrimiento noticioso de la migración venezolana están relacionados ( $\mathrm{p}$. 961).

Shore, Shore \& Abelló (1994) mencionan una gama de acciones que buscan respuesta y que engloban acciones de intolerancia, considerando el término de "chovinismo" que proviene del francés y que significa "exaltación desmesurada de lo nacional frente a lo extranjero" (Real Academia Española, 2021); además del término racismo, el cual debemos tener presente como una ideología, manifiesta bien como destrucción en masa hacia otro grupo étnico o racial, como una jerarquía de dominación entre razas o, una "acción social llevada a cabo por movimientos sociales o comunitarios de tipo nacionalista o religiosos" (Herranz, 2008, 32).

\begin{abstract}
El incremento del racismo y el chovinismo cultural, con la proliferación de ataques incendiarios contra refugios de inmigrantes, mezquitas $y$ sinagogas, la profanación de cementerios judíos, el creciente respaldo en favor de los partidos de extrema derecha o neonazis en Alemania, Francia, Bélgica e Italia, y la creciente oleada de nacionalismo, antisemitismo y hostilidad étnica en gran parte de la Europa del Este y la antigua Unión Soviética como manifestaciones más visibles (Shore, Shore \& Abelló, 1994, p. 32).
\end{abstract}

Por otra parte, es importante señalar que la profunda crisis política y social que se ha gestado en Venezuela ha generado un éxodo masivo de población de este país. según la OIM en el año 2020 el número de venezolanos que ingresaron a los países de América del Sur aumentó de 402 manera significativa. En la gráfica subsiguiente se evidencian estas cifras:

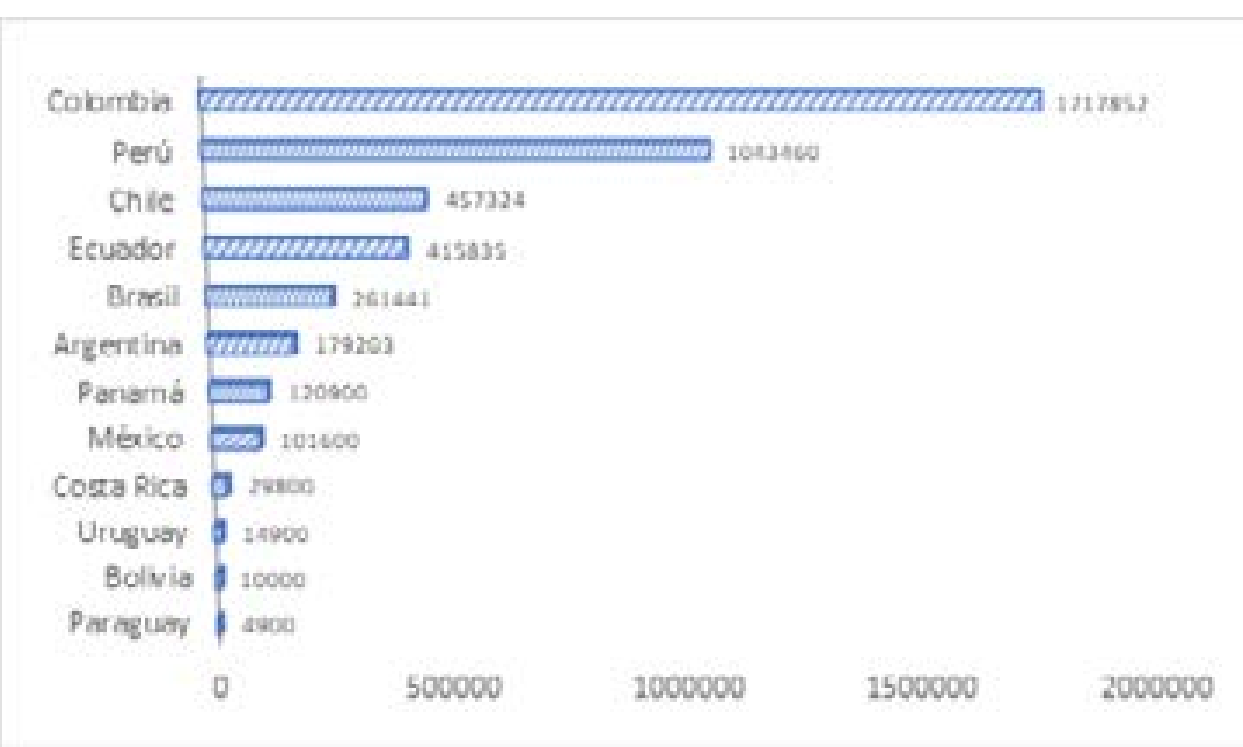

Gráfica 1. Población venezolana que ingresó a países latinoamericanos

Fuente: Elaboración propia 
Como se muestra en la gráfica anterior, Colombia ha sido uno de los países con el mayor número $(1.717,852)$ de recepción de migrantes venezolanos. Según el Grupo Interagencial sobre Flujos Migratorios Mixtos (GIFMM, 2020), la distribución de esta población en Colombia se encuentra ubicada de la siguiente manera:

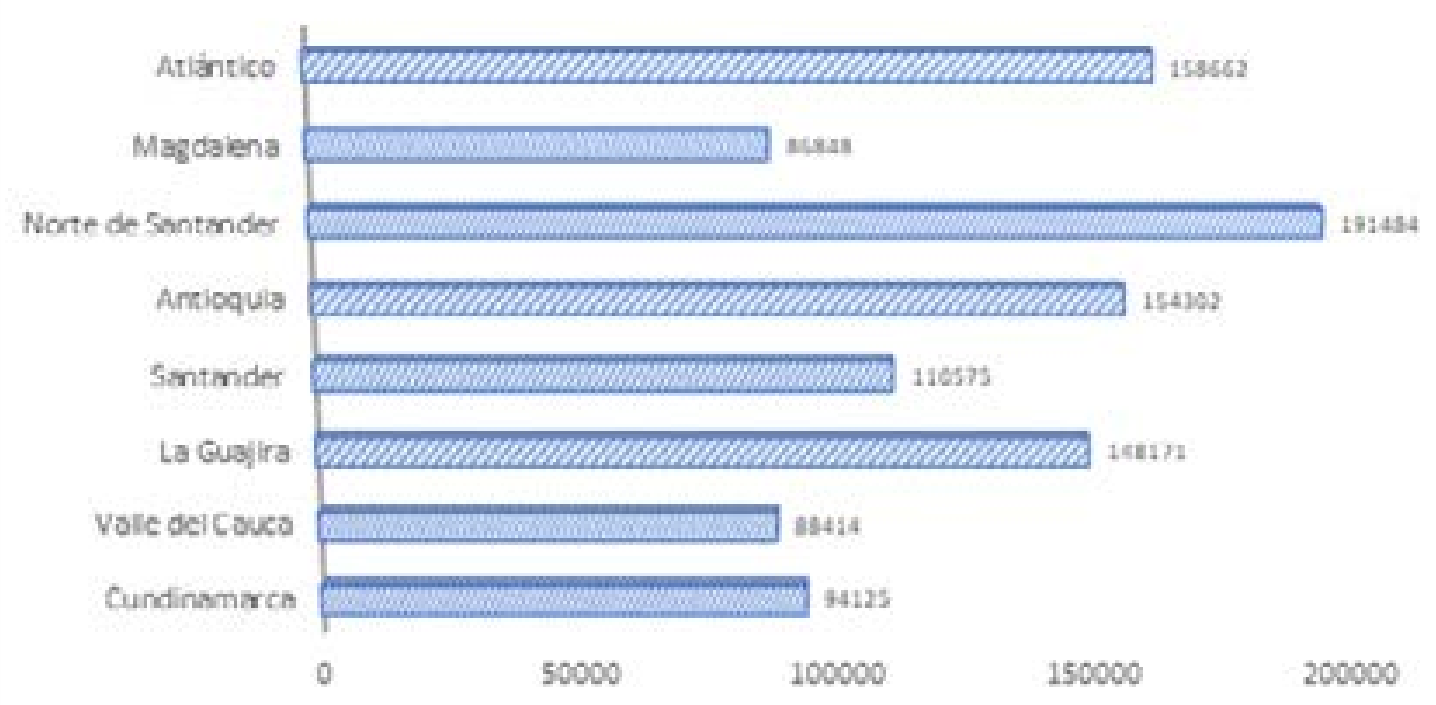

Gráfica 2. Recepción de migrantes por Departamento en Colombia

Fuente: Elaboración propia

Por este gran volumen de población venezolana asentada en Colombia se generan fenómenos sociales sensibles como la inseguridad en las diferentes ciudades, que aunque se ha comprado que ha aumentado, este aumento de la inseguridad está relacionado con el aumento poblacional que generó la migración, es decir, los delitos se han cometido también en contra de migrantes venezolanos "endilgar el deterioro de la seguridad en la ciudad a los migrantes y no a una gestión insuficiente, confunde a la ciudadanía y denota una preocupante falta de humildad para corregir los errores." (Mantilla, 2020, párr. 25), si bien es cierto que los migrantes se han visto involucrados en crímenes de los grupos armados al margen de la ley, narcotraficantes, y demás criminales que adquieren mano de obra joven y cualificada transitando las "trochas" (Duran, Rivera y
Castillo, 2019), a lo cual propone Santamaría (2020), que es "necesario desarrollar una política pública de seguridad integral que articule el trabajo tanto del sector defensa como de las entidades gubernamentales, y así mitigar el riesgo frente a la incorporación de población venezolana a grupos al margen de la ley" (p. 4).

La situación irregular en la que se encuentra la población venezolana al ingresar por medio de pasos ilegales a Colombia, genera, entre otras cosas, que las personas inmigrantes venezolanas en Colombia no tengan fácil acceso a los bienes y servicios que demandan como humanos. Por ende, la mendicidad ha sido una opción más para subsistir en un país ajeno, creando una cultura e idiosicracia en los niños migrantes que no es la más adecuada para el desarrollo de sus habilidades; así, se presenta 
una nueva problemática: "el uso de menores de edad dentro del contexto de mendicidad." (Valencia, Wayman, Newball y Henao, 2020, p.6). Aunque se debe hacer un enfoque especial de esta problemática, ya que

la mendicidad no es un delito y que se debe diferenciar lo que se entiende por mendicidad propia y por mendicidad ajena. El primer caso se refiere a las personas que piden dinero o ayuda en las calles por necesidad. El segundo, en cambio, se considera como una forma de trata de personas (Pungiluppi y Cortés citadas en Personería De Bucaramanga y Secretaría De Integración Social, 2019, p.90).

Aún así, se es consciente de que mendigar es una opción para los inmigrantes venezolanos en Colombia, aunque no se tienen demasiados estudios cuantitativos sobre cifras respecto a población migrante venezolana mendigando en Colombia son tantas familias venezolanas mendigando, pero no hay un organismo que haya hecho un registro de personas en esa condición (Moreno, 2020).

La prostitución también puede ser un indicador de discriminación hacía los inmigrantes venezolanos en Colombia en la medida que, como afirman Palacios, Torres, Luna y Rojas (2020) en su estudio se evidencia que

Se destacan características que reflejan ciertos estereotipos $y$ escenarios de discriminación similares en las cuatro ciudades, por ejemplo, el ejercicio de la prostitución o el imaginario de que las niñas, adolescentes y mujeres venezolanas se enfocan en temas sexuales y son quebrantadoras de relaciones intrafamiliares; este aspecto contribuye a la construcción de prejuicios contra las migrantes, incluso por parte de las mismas autoridades, y en ocasiones conduce a que sean víctimas de discriminación, explotación o trata de personas (párr. 11).

Así mismo, los mismos autores destacan que "Es una constante también el reconocimiento de la existencia de estigmas alrededor de la concepción de la mujer como objeto sexual (cosificación), lo cual puede derivar en escenarios de trata de personas." (Palacios, Torres, Luna y Rojas, 2020, párr. 10). Es así como se ven expuestas de igual forma en dignidad y en salud, ya que "Estas migrantes están en riesgo de sufrir violencia durante el ejercicio del acto sexual y se ven expuestas a infecciones de transmisión sexual (ITS) como sífilis y VIH, cuya prevalencia ha aumentado en áreas de alta concentración de población migrante" (p. 1307 - 1308). Sumado a todo lo anterior, cabe mencionar que dicha situación de migrantes buscando refugió y estabilidad económica en Colombia, y que encuentran una opción desesperada, que sólo puede lastimarlas a ellas, si sus familiares no se enteran, o porque por lo menos ellos mantienen su integridad y salud en buen estado. Las migrantes venezolanas trabajadoras sexuales ofrecen precios menores a los impuestos por las colombianas, lo que lleva a disputas por las zonas de trabajo en las calles de las grandes ciudades; "la corte constitucional colombiana decretó que las trabajadoras sexuales venezolanas tienen derecho a solicitar una visa de trabajo, lo que ayudó a frenar las deportaciones masivas tras ser detenidas por agentes de la ley" (Cada vez más venezolanas, 2017, párr.10).

Estas dinámicas sociales, al parecer generan en los residentes de las diferentes ciudades, actitudes y sentimientos de rechazo a la población migrante que con el tiempo se configuran en actitudes xenofóbicas. Este tema, es de 
interés particular de grupos de investigación y académicos de diferentes campos.

Así, existen estudios que revelan que en Colombia el rechazo a la población migrante proveniente de Venezuela, se sugiere en escenarios como los medios de comunicación (Cortés, 2018), donde, según entienden Ordoñez y Ramírez (2018), las políticas proclamadas en favor de los migrantes venezolanos "van de la mano con mensajes contradictorios producidos en los medios que, en últimas, le presentan al público a una población indiferenciada que pone en peligro al cuerpo de la nación." (p. 48).

Alrededor del mundo y de la historia se han presentado obstáculos para un aprovechamiento de la mano de obra extranjera y un bajo apoyo a población migrante de encontrar una remuneración económica con la cual subsistir.

\begin{abstract}
los países de destino 0 receptores se muestran cada vez menos dispuestos a acoger a los trabajadores inmigrantes, situación que se expresa en la colocación de nuevos y mayores obstáculos burocráticos, pero también mediante el despliegue de cuerpos de seguridad en zonas fronterizas, el cierre de fronteras o el levantamiento de muros físicos que permitan frenar y desincentivar los flujos migratorios (Pineda \& Ávila, 2019, p. 53).
\end{abstract}

Es por esto que otro escenario de discriminación contra el migrante es el campo laboral donde "Alrededor del $66 \%$ de los entrevistados declaró que se encontraba sin empleo o que estaba trabajando de manera informal [...]" (La Oficina del Alto Comisionado de las Naciones Unidas para los Refugiados [ACNUR], 2019). Dichas cifras muestran una mediana cobertura en empleabilidad para migrantes venezolanos en Colombia, además de que se desvaloriza su trabajo por la necesidad, afectando esto también a los trabajadores colombianos y favoreciendo únicamente a actores políticos y económicos "estas condiciones de vulnerabilidad de los migrantes

venezolanos han acabado siendo rentables para diversos actores políticos y económicos, quiénes obtienen múltiples ganancias gracias a este drama humano." (Pineda \& Ávila, 2019, p.91).

Respecto a la preparación académica, la población venezolana tiene niveles incluso más altos que la población colombiana "esto se debe a que en Venezuela hay acceso gratuito a la educación hasta tercer nivel." (Ramírez, Linares y Useche, 2019, p.31). Aunque muchos niños y niñas se encuentran en una situación difícil respecto a su preparación académica arraigada por la migración, esto en el caso específico de Colombia, se ha orientado de la siguiente manera:

se puede afirmar que el Gobierno colombiano ha adelantado labores y diseñado estrategias aplicadas a la necesidad de integración de los migrantes venezolanos, sin embargo, es evidente que aún falta focalizar aún más en materia de interculturalidad dentro de la estructura y sistema educativo. Por lo cual, tanto el acceso, focalización y nuevas oportunidades proyectan un buen futuro de acción, al menos en diseño de Política Pública. En cuanto al acceso se cumplen 6/6 (25\%), necesidad de focalización $4 / 5(20 \%)$, nuevas oportunidades $3 / 5$ (15\%), educación intercultural para todos $1 / 5(5 \%)$. Por lo que, desde la política pública, Colombia está integrando en un $65 \%$ en materia educativa a los estudiantes migrantes provenientes de 
Venezuela. (Pinto, Amaya y Sáenz, 2019, párr. 60).

Por el fenómeno descrito se hace necesario indagar sobre las percepciones que tienen los jóvenes escolarizados sobre la migración venezolana hacia Colombia y detectar posibles actitudes xenofóbicas que puedan en un futuro generar conflictos en las instituciones educativas.

\section{METODOLOGÍA}

Esta investigación adopta un enfoque cuantitativo a nivel descriptivo utilizando el muestreo no probabilístico para la selección de los informantes, bajo la técnica de vinculación voluntaria. El instrumento utilizado corresponde al cuestionario propuesto en la investigación de Civalero, Alonso y Brussino (2019) que se deriva de la adaptación al castellano propuesta por Rueda y Navas (1996) y Cárdenas et al. (2007), trabajos que a su vez se originan de la Escala de Prejuicio Sutil y Manifiesto de Pettigrew \& Meertens (1995). A este cuestionario se le realizó revisión de la redacción de los ítems y los investigadores fungieron como pares evaluadores en el proceso de validación de contenido al tiempo que se ajustaban al contexto regional.

La muestra quedó integrada por un total de 616 adolescentes ambos sexos con predominio del género femenino (54.2\%). Estas personas estuvieron matriculadas en los grados Décimo y Undécimo durante el año 2020 en diversas instituciones educativas de un municipio fronterizo con Venezuela. Se resaltan las características de los integrantes de la muestra en el hecho que hacían parte de una iniciativa de la Alcaldía Municipal cuyo objetivo era determinar en los adolescentes del municipio cualquier indicio de rechazo hacia el flujo constante de migrantes y si percibían en ellos cualquier indicio de amenaza. Por tal motivo, se consideraron estudiantes de colegios tanto públicos como privados.

Como ya se mencionó los integrantes de la muestra fueron quienes de forma voluntaria aceptaron la invitación a ser parte de esta investigación, tras haber recibido la invitación a diligenciar el link de Google Form previo consentimiento de los rectores de dichas instituciones educativas y previo proceso de sensibilización sobre la importancia de sus opiniones para la generación de programas de intervención a futuro. Cada participante debió responder el cuestionario que estaba compuesto de dos secciones, por un lado, se encontraban las variables asociadas con su perfil demográfico y académico, y, por otra parte, se ubicaban 20 afirmaciones con formato de respuesta en escala Likert con cinco opciones de respuesta que pretender determinar el nivel de acuerdo o desacuerdo con cada una de ellas. La Tabla 1 evidencia la desagregación de la Escala de Prejuicio utilizada por Civalero, Alonso y Brussino (2019).

Tabla 1. Descripción de la Escala de Prejuicio Manifiesto y Sutil

\begin{tabular}{clc}
\hline Tipo de Prejuicio & \multicolumn{1}{c}{ Dimensión de análisis } & Número de ítems \\
\hline \multirow{2}{*}{ Manifiesto } & Amenaza y Rechazo & 6 \\
\cline { 2 - 3 } & Relaciones Cercanas & 4 \\
\hline \multirow{2}{*}{ Sutil } & Valores Tradicionales & 4 \\
\cline { 2 - 3 } & Diferencias Culturales & 4 \\
\cline { 2 - 3 } & Emocionales Positivas & 2 \\
\hline
\end{tabular}


Finalmente, los datos recolectados son descargados en un archivo de Excel para luego ser exportados al SPSS, software estadístico con el que posteriormente se realizan los análisis descriptivos que permiten ir conociendo la opinión de los adolescentes. Se resalta que estos estudiantes corresponden a los cinco primeros días de medición y no representa el total de informantes, es decir, que corresponden a resultados parciales dado que la información general corresponde al ente municipal encargado de la investigación.

\section{HALLAZGOS Y DISCUSIÓN}

En cuanto a las características demográficas de los estudiantes que participaron de este trabajo se puede observar por medio de la tabla 2 el $54.2 \%$ de los estudiantes integrantes de la muestra son de genero femenino. En cuanto al grado que cursan actualmente, el $61.4 \%$ se encuentran en grado décimo; respecto al tipo de institución educativa, el $70.9 \%$ estudian en instituciones educativas de orden público y en cuanto al estrato socioeconómico, el $87.7 \%$ residen en viviendas o barrios cuyas características corresponden a las de los estratos uno y dos.

Tabla 2. Características demográficas de los integrantes de la muestra

\begin{tabular}{ccc}
\hline Característica & Niveles de respuesta & Porcentaje \\
\hline \multirow{3}{*}{ Género } & Femenino & $54.2 \%$ \\
\cline { 2 - 3 } & Masculino & $45.8 \%$ \\
\cline { 2 - 3 } Grado académico que cursa & Total & $\mathbf{1 0 0 . 0 \%}$ \\
\cline { 2 - 3 } & Décimo & $61.4 \%$ \\
\cline { 2 - 3 } & Undécimo & $38.6 \%$ \\
\hline \multirow{3}{*}{ Tipo de institución educativa } & Total & $\mathbf{1 0 0 . 0 \%}$ \\
\cline { 2 - 3 } & Pública & $70.9 \%$ \\
\cline { 2 - 3 } & Privada & 29.1 \\
\cline { 2 - 3 } Estrato Socioeconómico & Total & $\mathbf{1 0 0 . 0 \%}$ \\
\cline { 2 - 3 } & Estrato Uno & $35.9 \%$ \\
\cline { 2 - 3 } & Estrato Dos & $51.8 \%$ \\
\cline { 2 - 3 } & Estrato Tres & $12.3 \%$ \\
\hline
\end{tabular}

\section{ANÁLISIS DEL TIPO DE PREJUICIO}

A pesar de que la escala valorativa correspondía a una escala Likert con cinco niveles siendo una variable de tipo ordinal, se puede realizar con ellas un tratamiento como si fueran variables cuantitativas. A partir de este razonamiento, se puede determinar el promedio general de cada tipo de prejuicio ello con el fin de identificar la transparencia con que los muchachos manifiestan su postura ante la constante llegada de migrantes venezolanos a su contexto local.
Se pudo determinar que el promedio obtenido por la categoría de prejuicio sutil está por encima del valor medio de la escala valorativa y supera a la categoría de prejuicio manifiesto en más de cinco décimas. Ello podría ser evidencia de rechazo hacia la llegada masiva de ciudadanos venezolanos al contexto local, enmascarado u oculta bajo un comportamiento de aceptación. 


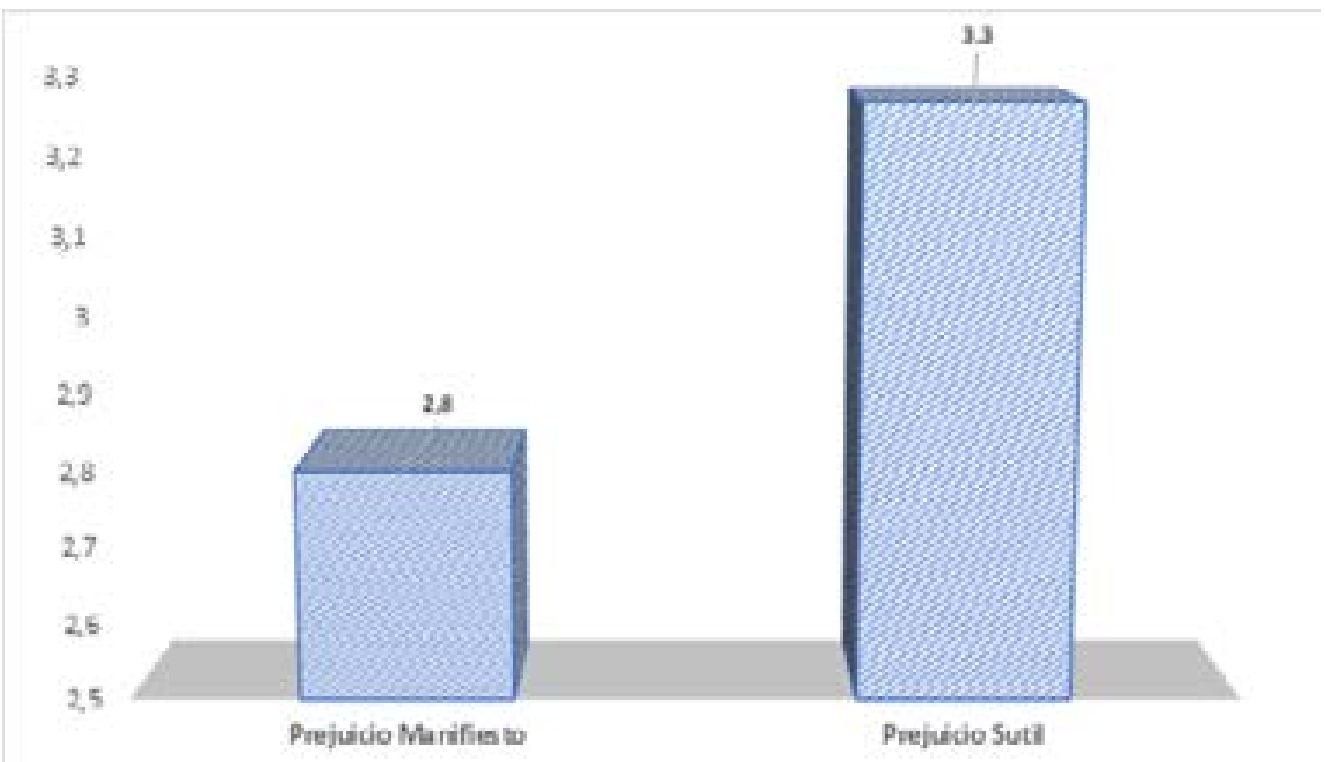

Figura 1. Calificación promedio para cada categoría de prejuicio

Fuente: Elaboración propia

\section{PREJUICIO MANIFIESTO}

Al realizar un comparativo entre cada una de las dimensiones del prejuicio Manifiesto, se pudo determinar tal como se aprecia en la Figura 2, que dimensión denominada Relaciones Cercanas poseen el promedio más bajo dado que en opinión de los estudiantes encuestados rechazan el intimar con inmigrantes de forma personal siendo una postura más radical en las mujeres (promedio de 1.14), dado que los hombres no descartan esta opción (promedio de 3.84).

Estudios como el Cárdenas, Gómez, Méndez y Yáñez (2011) reportan resultados similares a los mostrados en este informe afirmando que las mujeres en algunos casos manifiestan mayores niveles de prejuicio manifiesto hacia los inmigrantes, sin embargo, es importante contrastar de una manera más profunda el tipo de muestra, es decir, estrato socioeconómico, edad y contexto, puesto que por ejemplo los estudios realizados con mujeres de grupos feministas (De Freitas y López, 2017) se muestran que las mujeres son más tolerantes y no presentan promedios altos de prejuicios manifiestos

En lo respecta, a aceptar la consolidación de una relación de alguno de sus familiares con un ciudadano inmigrante, se determinó que, a nivel general, existe una postura de rechazo en este sentido sin importar si el ciudadano inmigrante cuenta con recursos económicos para garantizar esta unión. Se rechaza de forma categórica debido a que consideran, esta unión debilitaría las características propias de nosotros como colombianos, puesto que existe el imaginario que el ciudadano venezolano es muy flojo para trabajar.

Finalmente, en cuanto si aceptarían que un ciudadano venezolano bien preparado asumiera el rol de docente dentro de su proceso de formación pedagógica, se obtuvo un promedio de 2.14 lo cual pone de manifiesto una postura de rechazo.

Continuando el análisis con la dimensión Amenaza o Rechazo, se evidenció rechazo en los ítems 3 y 6 con promedio de 2.45 y 2.74 
respectivamente, los cuales corresponden a que los inmigrantes han llegado al país a esperar recibir ayudas sociales o económicas sin afanarse por buscar un empleo (tal como lo hace la mayoría de ciudadanos colombianos), por lo que consideran son personas deshonestas y poco confiables.

Los estudiantes encuestados en promedio aseguran les resulta indiferente (con promedio de 3.05) si los ciudadanos se sienten cómodos en nuestro país y de forma categórica, cuestionan la forma como la clase política del país se ha dedicado a solucionar los problemas de esta población y ha descuidado satisfacer las necesidades de los nacionales (promedio de 4.68).
En esta línea argumentativa, los estudiantes encuestados resaltan que los inmigrantes han llegado al país a ocupar las escasas fuentes de empleo (con promedio de 3.86) que hay en la ciudad, pero debido a sus pocas competencias laborales luego son despedidos. Esta situación se hace recurrente por lo que termina afectando la vinculación laboral de ciudadanos colombianos.

Estas percepciones al parecer se exteriorizan, no solo por la inserción de la mano de obra venezolana menor remunerada que la colombiana, si no por la profunda crisis económica que vive la ciudad y el departamento (Barrientos, Berbesí, Contreras y Lesmes, 2019).

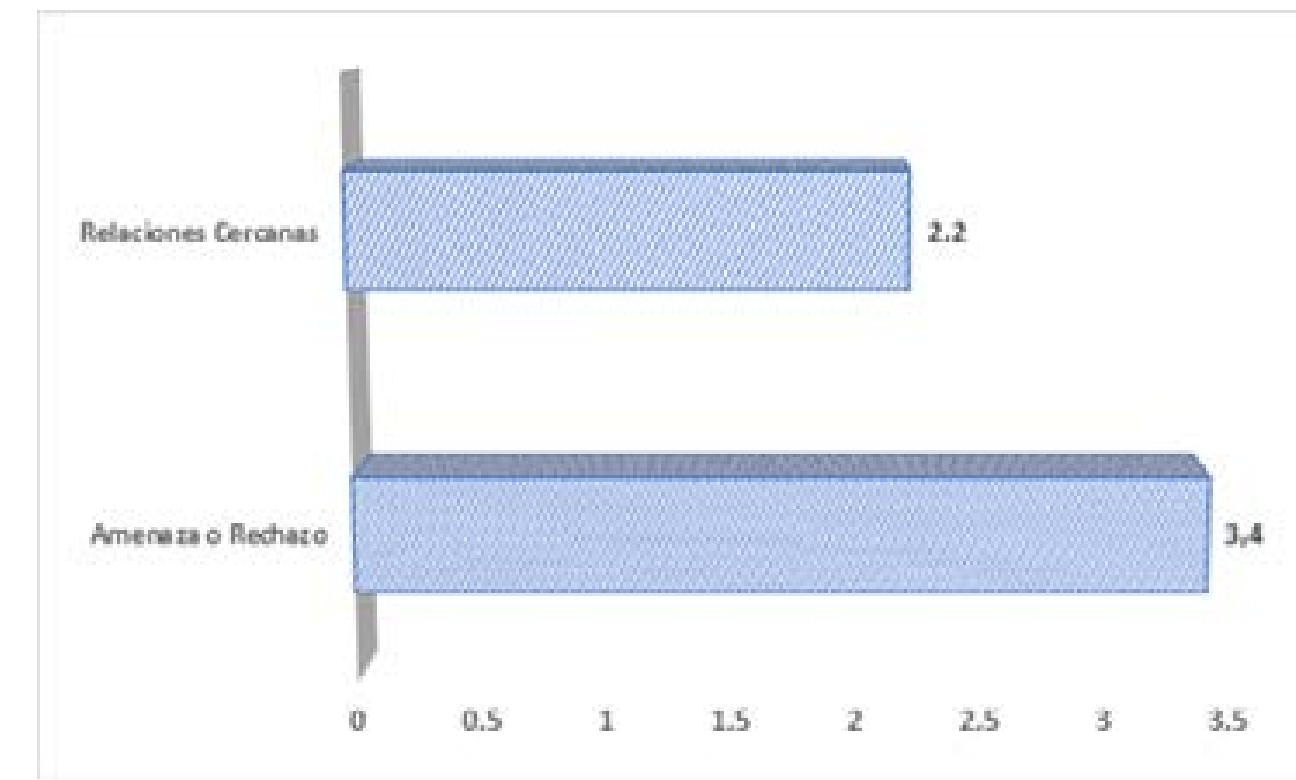

Figura 2. Calificación promedio para cada una de las dimensiones del Prejuicio Manifiesto

Fuente: Elaboración propia

\section{PREJUICIO SUTIL}

Se analiza cada una de las dimensiones de esta categoría de prejuicio, se determinó que los ítems en lo que se resaltan las Diferencias Culturales se destacaron con mayor aceptación entre los estudiantes encuestados con un promedio de 4.01, sobresaliendo como principales aspectos diferenciadores las creencias y prácticas religiosas con un promedio de 4.88 y el dialecto utilizado con 4.81 puntos en promedio. Continuando con el estudio de los ítems de esta dimensión, los estudiantes aseguran que la forma en que los padres inmigrantes educan a sus hijos es muy diferente a la crianza en nuestro país y región, destacando que tienen más libertades y por ello es fácil que se desvíen del camino correcto. Como último elemento 
de esta dimensión, les resulta indiferente las prácticas sexuales empleadas por esta población inmigrante.

Con respecto a estas dimensiones es importante citar un análisis que realiza Cisneros (2001), cuando afirma "que la xenofobia se alimenta de prejuicios nacional que son históricos y culturales. Tanto xenofobia y como el racismo tienen como primera consecuencia la discriminación del otro y su segregación como sujeto absolutamente diferente" (p.187).

En cuanto a la dimensión Valores Tradicionales se observó un promedio general de 3.80 puntos, destacando que esta población migrante no debería esperar en nuestro país un trato especial para ellos, por el contrario, deberían esforzarse mucho más tal como lo hacen los nacionales y así podría alcanzar mejor calidad de vida, pero sería preferible que lo hicieran en su país y no en el nuestro, ya que ellos los perciben como invasores. Se ratifica lo mencionado anteriormente, los estudiantes encuestados manifiestan que los padres inmigrantes que han llegado a nuestro país les enseñan valores y costumbres diferentes a las nuestras para alcanzar el éxito, se resalta en ellos el facilismo con que deseo conseguir las cosas, tal como se resalta en Dolores Palacios et al. (2018) quienes resaltan la importancia del hogar y los valores que en él se promueven en el proceso de formación de los jóvenes y adolescentes.

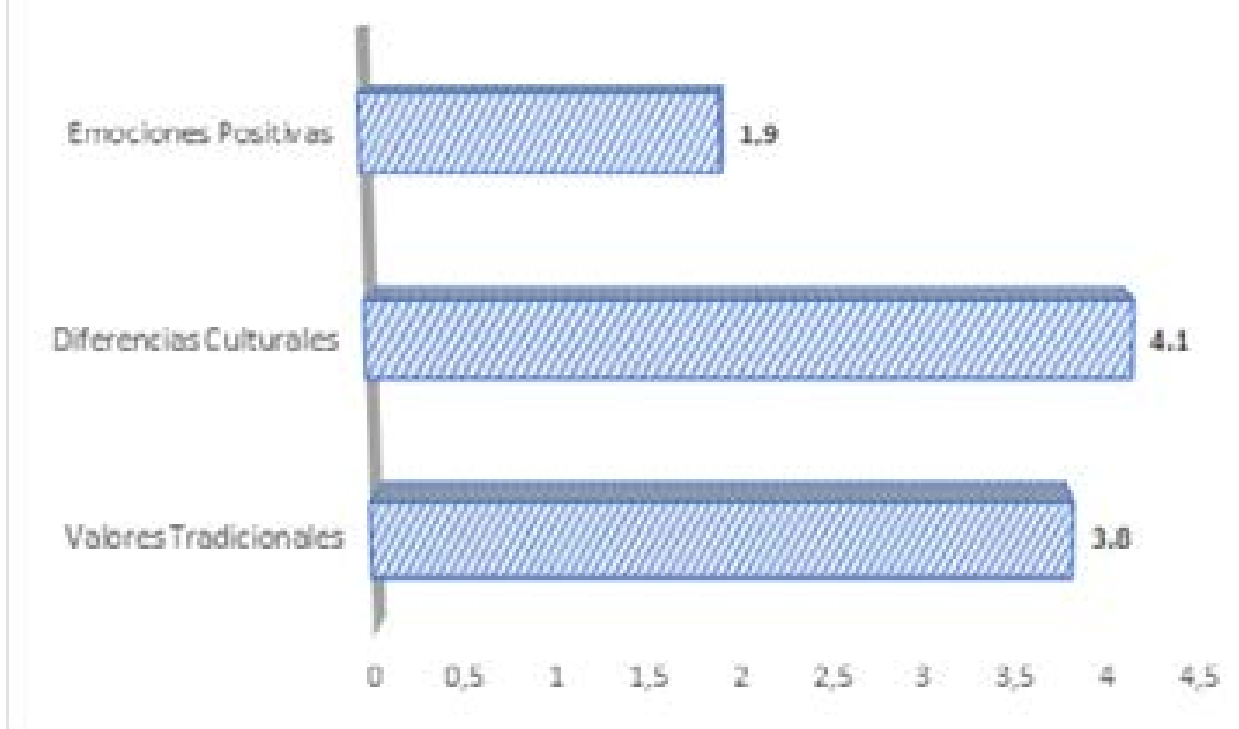

Figura 3. Calificación promedio para cada una de las dimensiones del Prejuicio Sutil

Fuente: Elaboración propia

Finalmente, en cuanto el análisis al interior de la dimensión Emociones Positivas se pudo determinar en los encuestados que rechazan con promedios de 1.68 y 2.14 los dos ítems de esta dimensión, en los que se manifestaba la admiración que podían sentir hacia los ciudadanos inmigrantes que han llegado al país y el sentimiento de solidaridad que podían experimentar hacia ellos, respectivamente.
Estos sentimientos de solidaridad pueden entenderse muy bien desde los enunciados de Penchaszadeh y Sferco (2019) cuando vehemente afirman que

Así, la solidaridad, entendida como práctica de reciprocidad social hacia aquellos que se consideran como iguales, no solo ha de diferenciarse de 
la hospitalidad-siempre marcada por una frontera, una llegada, un recibimiento, una extrañeza - sino también de otras prácticas, como ser: la caridad, que se ofrece al absolutamente desigual y el cuidado, que se profesa a los de la propia familia (Párr. 37)

En este sentido los encuestados aseguran que en un comienzo se sintieron solidarios ante la situación que estaban experimentando los ciudadanos venezolanos en su país y que en algún momento desde sus hogares les apoyaron con ayudas. Pero con el paso del tiempo esta situación se ha salido de control y son percibidos como una carga para el país y es muy poco lo que ellos aportan, por ello se manifiesta de forma tenue e indirecta ese sentimiento de rechazo hacia la permanencia de estas personas en nuestra región y país.

\section{CONCLUSIONES}

El estudio analiza dos dimensiones importantes: el prejuicio manifiesto y el prejuicio sutil, en cuanto al primero se concluye que se evidencia un rechazo radical entablar relaciones cercanas hacia la población migrante tales como la relación de alguno de sus familiares con un ciudadano inmigrante puesto que puede debilitar la estabilidad familiar y económica del hogar, según los argumentos de los encuestados.

Así mismo se pudo determinar que lo migrantes son considerados una amenaza desde la perspectiva de los encuestados puesto que llegaron en su éxodo al país con el objetivo de recibir ayuda de tipo social y económica quitando oportunidades a la población colombiana Es importante señalar que el prejuicio manifestó se evidencia de una manera más radical entre el género femenino que el masculino, donde se observa una mayor tolerancia al migrante por parte de los hombres que hicieron parte de la investigación.
Con respecto al prejuicio sutil se encontró que hay cierto rechazo a los elementos culturales $y$ religiosos de los migrantes siendo más evidente los relacionados con el dialecto utilizado y las formas de educación de padres a hijos.

No obstante, de estos resultados donde evidentemente se presentan prejuicios de tipo manifiesto y sutil, también se evidencian percepciones de solidaridad, empatía y tolerancia poniendo como plataforma ética la igualdad humana y la preocupación por el otro.

\section{REFERENCIAS BIBLIOGRÁFICAS}

Agencia de las Naciones Unidas para los Refugiados. (2019). Un estudio de ACNUR destaca los riesgos que enfrentan las personas venezolanas vulnerables en movimiento. Recuperado de: https://www.acnur.org/noticias/ briefing/2019/7/5d31abd74/un-estudio-deacnur-destaca-los-riesgos-que-enfrentan-laspersonas-venezolanas.html

Bouza, F. (2002). Xenofobia. Glosario para una Sociedad Intercultural. Recuperado de: https:// www.ucm.es/data/cont/docs/471-2013-11-05xeno3.pdf

Barrientos-Rosales, M. D. L. Á., Berbesí-Carrillo, W. O., Contreras-Suárez, G., \& Lesmes-Silva, A. K. (2019). Incidencia de la economía informal en la movilidad en la ciudad de Cúcuta. Revista CONVICCIONES, 6(11), 101-105.

Cardenas, M., Gómez, F., Méndez, L. y Yáñez, S. (2011). Reporte de los niveles de prejuicio sutil y manifiesto hacia los inmigrantes bolivianos y análisis de su relación con variables psicosociales. Psicoperspectivas, 10 (1), 125-143. Doi: http://dx.doi.org/10.5027/ psicoperspectivas-Vol10-Issue1-fulltext-134

Civalero, L., Alonso, D., \& Brussino, S. (2019). Evaluación del prejuicio hacia inmigrantes: adaptación argentina de la escala de prejuicio 
sutil y manifiesto. Ciencias Psicológicas, 13(1), 119-133.

Cortés, C. (2018). Xenofobia y periodismo: Colombia y la migración venezolana. PalabraClave, 21(4), 960-963. DOI: 10.5294/ pacla.2018.21.4.1

Cárdenas, M., Music, A., Contreras, P., Yeomans, H., \& Calderón, C. (2007). Las nuevas formas de prejuicio y sus instrumentos de medida. Revista de Psicología, 16(1), 69-96

Chavarría, F. T. (1994). Sociopatología de la xenofobia y de los nacionalismos. Papers: revista de sociología, (43), 139-147. DOI: http:// dx.doi.org/10.5565/rev/papers/v43n0.1734

Cisneros, I. H. (2001). Intolerancia cultural: racismo, nacionalismo, xenofobia. Perfiles latinoamericanos: revista de la Facultad Latinoamericana de Ciencias Sociales, Sede México, 10(18), 177-189.

Cada vez más venezolanas desesperadas recurren a la prostitución en Colombia. (27 de julio de 2017). Infobae. Recuperado de: https://www.infobae.com/america/ venezuela/2017/07/27/the-economist-masvenezolanas-desesperadas-recurren-a-laprostitucion-en-colombia/?outputType=amptype

De Freitas, L. F. R., \& López, L. P. M. (2017). Sobre feminismo, sobre racismo, sobre xenofobia, sobre tudo: desequilíbrios narrativos em performances heterossexuais de um aluno migrante branco. Calidoscópio, 15(2), 305-316. Doi: doi: 10.4013/cld.2017.152.08

Dolores Palacios, M., Conforme-Zambrano, E. G., Villavicencio, F., Arpi, N., Clavijo Castillo, R., \& Mora, J. C. (2018). Manifestaciones de control de padres y madres de familia en Cuenca, Ecuador, sobre sus hijos de entre 5 y 12 años. Revista Perspectivas, 3(1), 44-58. https://doi. org/10.22463/25909215.1423
Duran, A., Rivera, J., y Castillo, J. (2019, 21 de diciembre). Flujos migratorios de venezolanos y comportamiento delictivo: la necesidad de política pública para prevenir la estigmatización, marginalización y criminalización. Revista Zero. Recuperado de: https://zero.uexternado.edu.co/flujosmigratorios-de-venezolanos-y-comportamientodelictivo-la-necesidad-de-politica-publica-paraprevenir-la-estigmatizacion-marginalizacion-ycriminalizacion/

Fernández, F. (2004). Análisis crítico sobre las migraciones procedentes de Perú y Bolivia: el discurso periodístico de El Mercurio y La Tercera. Si somos americanos. Revista de Estudios Transfronterizos, 6(5), 123-148. Recuperadodehttps://www.sisomosamericanos. $\mathrm{cl} / \mathrm{index} . \mathrm{php} / \mathrm{sisomosamericanos/article/}$ view/512

Gaborit, M. (2020). La construcción social de la persona migrante como enemigo. In García C. (Ed.), Puentes, no muros: Contribuciones para una política progresista en migraciones (pp. 1-24). Ciudad de Buenos Aires, Argentina: CLACSO. DOI:10.2307/j.ctv1gm022b.6

Herranz, G. (2008). Xenofobia: un estudio comparativo en barrios y municipios almerienses. RevistaEspañolade Investigaciones Sociológicas (Reis), 121(1), 107-132. Recuperado de: https://www.ingentaconnect. com/content/cis/reis/2008/00000121/00000001/ art00004

Moreno, M. (2020). Mendicidad, opción de vida de decenas de venezolanos en Guayaquil.

El Universo. Recuperado de: https://www. eluniverso.com/guayaquil/2020/11/07/ nota/8040289/mendicidad-opcion-vidadecenas-venezolanos/

Ordóñez, J y Ramírez, H. (2019). (Des)orden nacional: la construcción de la migración venezolana como una amenaza de salud y 
seguridad pública en Colombia. Rev. Cienc Salud. 17, 48-68. Doi: http://dx.doi.org/10.12804/ revistas. urosario.edu.co/revsalud/a.8119

Palacios, M., Torres, M., Luna, B., y Rojas, N. (2020). La migración femenina en Colombia: experiencias locales para el abordaje de la trata de personas. Revista Zero. Recuperado de: https://zero.uexternado.edu.co/la-migracionfemenina-en-colombia-experiencias-localespara-el-abordaje-de-la-trata-de-personas/

Personería de Bucaramanga y Secretaría del Interior (2019). ¿Explotación o necesidad?: la explicación pendiente sobre los niños mendigos migrantes. Recuperado de: https://migravenezuela.com/web/articulo/ denuncias-sobre-mendicidad-infantil-enbucaramanga/1375

Pineda, E., \& Ávila, K. (2019). Aproximaciones a la Migración Colombo-venezolana: Desigualdad, Prejuicio y Vulnerabilidad. Revista Misión Jurídica, 12, 46 - 97. Recuperado de: https:// revistes.ub.edu/index.php/clivatge/article/ view/28596

Pinto, L., Amaya, P., y Sáez, F. (2019). La integración de los venezolanos en Colombia en los ámbitos de la salud y la educación. Espacio abierto: cuaderno venezolano de sociología, 28(1), 199-223. Recuperado de: https://www.redalyc.org/ jatsRepo/122/12262976013/html/index.html

Pettigrew, T. F., \& Meertens, R. W. (1995). Subtle and blatant prejudice in Western Europe. Europeanjournal of social psychology, 25(1), 5775. Doi: $10.1002 /$ ejsp.2420250106.

Rueda, J. F., \& Navas, M. (1996). Hacia una evaluación de las nuevas formas del prejuicio racial: las actitudes sutiles del racismo. Revista de Psicología Social, 11(2), 131-149. Doi: $10.1174 / 02134749660569314$.
Penchaszadeh, Ana Paula y Sferco, Senda Inés. (2019). Solidaridad y Fraternidad. Una nueva clave ético-política para las migraciones. REMHU: Revista Interdisciplinar da Mobilidad Humana, 27 (55). Doi: https://doi. org/10.1590/1980-85852503880005510

Real Academia Española. (2021). Chovinismo. Recuperado de: https://dle.rae.es/chovinismo

Ramírez, J., Linares, Y., y Useche, E. (2019). Geopolíticas migratorias, inserción laboral y xenofobia: migrantes venezolanos en Ecuador. En Cécile Blouin Después de la Llegada. Realidades de la migración venezolana. Lima, Perú: Themis-PUCP. Recuperado de: https:// www.aacademica.org/jacques.ramirez/22

Stavenhagen, R., \& Salinas, S. (1994). Racismo y xenofobia en tiempos de la globalización. Estudios Sociológicos, 12(34), 9-16. Recuperado de: https://www.jstor.org/stable/40420254?readnow=1\&refreqid=excelsior\%3Ad3d7190cd54ef1 $896039601 \mathrm{fdf} 32 \mathrm{a} 27 \mathrm{e} \& \mathrm{seq}=2 \#$ page_scan_tab_ contents

Shore, C., Shore, C., \& Abelló, M. (1994). Etnicidad, xenofobia y las fronteras de Europa. Historia Y Fuente Oral, (12), 3141. Recuperado de: http://www.jstor.org/ stable/27753443

Vallejo, M., Mesa, L., Melgarejo, Z., \& Llanes, M. (2020). Migración, prostitución y salud en territorios de frontera. El caso de las mujeres venezolanas en situación de prostitución en la frontera norte de Colombia. En M. Fernández., J, Gutiérrez., A, Herrera., M, Martínez., K, Pérez, (Eds.), Libro de Actas Akten Liburua Conference Proceedings (1305 - 1333). España: REEDES.

Valencia, Y., Newball, W., Wayman, N., \& Henao, D. (2020). Utilización de la niñez venezolana dentro del contexto de mendicidad. Recuperado de: : http://hdl.handle. net/10946/4807 